\title{
First Positron Annihilation Lifetime Measurement of $\mathrm{Pu}$
}

\author{
C. Colmenares \\ R.H. Howell \\ D. Ancheta \\ T. Cowan \\ J. Hanafee \\ P. Sterne
}

This is an informal report intended primarily for internal or limited external distribution. The opinions and conclusions stated are those of the author and may or may not be those of the Laboratory.

Work performed under the auspices of the U.S. Department of Energy by the Lawrence Livermore National Laboratory under Contract W-7405-ENG-48. 


\section{DISCLAIMER}

This document was prepared as an account of work sponsored by an agency of the United States Government. Neither the United States Government nor the University of California nor any of their employees, makes any warranty, express or implied, or assumes any legal liability or responsibility for the accuracy, completeness, or usefulness of any information, apparatus, product, or process disclosed, or represents that its use would not infringe privately owned rights. Reference herein to any specific commercial product, process, or service by trade name, trademark, manufacturer, or otherwise, does not necessarily constitute or imply its endorsement, recommendation, or favoring by the United States Government or the University of California. The views and opinions of authors expressed herein do not necessarily state or reflect those of the United States Government or the University of California, and shall not be used for advertising or product endorsement purposes.

This report has been reproduced directly from the best available copy.

Available to DOE and DOE contractors from the Office of Scientific and Technical Information

P.O. Box 62, Oak Ridge, TN 37831

Prices available from (615) 576-8401, FTS 626-8401

Available to the public from the

National Technical Information Service

U.S. Department of Commerce 5285 Port Royal Rd.,

Springfield, VA 22161 


\title{
First positron annihilation lifetime measurement of $\mathrm{Pu}$
}

\author{
Carlos Colmenares, Richard H Howell, Dione Ancheta, \\ Tom Cowan, Jim Hanafee, and Phil Sterne \\ Lawrence Livermore National Laboratory, Livermore CA 94550
}

We have made the first measurement of defects in an aged sample of $\delta$ phase, Ga stabilized $\mathrm{Pu}$, using positron annihilation lifetime spectroscopy. This measurement validates the procedure necessary to perform measurements on this highly toxic material and obtain data representative of sample conditions. Comparison of the positron annihilation lifetime analysis of the data with calculated values suggests that He filled vacancies or vacancy clusters dominate the defect population. Such defects are the necessary precursor to void growth and swelling.

The evolution of defects resulting from the radioactive decay of $\mathrm{Pu}$ during its life in the stockpile is one of the unknown quantities affecting our confidence in predictions of the limit on stockpile components. Radiation damage leads to changes in the size and strength of metals studied for reactor and accelerator use and similar effects may be expected in $\mathrm{Pu}$. The evolution of radiation produced vacancies into larger void structures and accompanying macroscopic swelling may occur in $\mathrm{Pu}$ at some age. A detailed understanding of the defects in self irradiated $\mathrm{Pu}$ is required to predict the time scale of void swelling and related radiation effects. We have made the first measurement of defects in an aged sample of $\delta$ phase $\mathrm{Pu}$ using positron annihilation lifetime spectroscopy.

Positron annihilation lifetime spectroscopy is an established tool for the analysis of vacancies and voids in metals and compounds. There are many techniques that can identify the species and size of impurities. There are few, however, that can detect open volume defects such as atomic vacancies or voids. All positron annihilation spectrographic methods are particularly sensitive to determining low concentrations of the class of defects including vacancies, voids and negatively charged defects. This sensitivity stems from the attractive interaction between these defects and positrons which often binds the positron to the defect site. Using positron annihilation lifetime spectroscopy determines the concentration and size or charge of defects in metals, semiconductors and molecular or organic compounds.

Positron annihilation lifetime spectroscopy measures the electron density at the annihilation site. Since the electron density is sensitive to the defect volume there is a distinct correlation between the positron lifetime and defect size. In metals and some compounds the correlation between lifetime and defect size can be calculated from first principles with sufficiently high accuracy to differentiate between major defect classes such as vacancies, vacancy clusters and voids. Defect 
concentrations can be determined from the fraction of positrons annihilating at the defect site.

A newly developed high energy positron beam and lifetime spectrometer, located in a facility capable of containing $\mathrm{Pu}$ material enabled our measurements. Our high energy beam is derived from a $100 \mathrm{mCi}^{22} \mathrm{Na}$ source moderated by a 2 micron thick tungsten single crystal foil positioned in the terminal of a $3 \mathrm{MV}$ Pelletron electrostatic accelerator. This beam contains a current of $5 \times 10^{5} \mathrm{e}^{+} \mathrm{s}^{-1}$ at 3 $\mathrm{MeV}$. LLNL is the only US facility to offer this capability.

To perform measurements, a sample of aged $\mathrm{Pu}$ was vacuum-encapsulated in the LLNL Pu facility in a holder with two 2 mil stainless steel windows for measurement. After transportation to the measurement facility, positrons accelerated in a beam to $3 \mathrm{MeV}$ were introduced into the capsule through the thin overlaying windows. Timing signals to form a positron annihilation lifetime distribution were obtained from the positrons as they were implanted and after annihilation.

We have performed calculations and experiments to design the sample capsule and to determine potentially useful configurations of beam and detectors for the lifetime measurement. Lifetime measurements with the Pelletron were conducted on $\mathrm{U}$ and other metals held in a capsule similar to the Pu capsule to determine the characteristics of the lifetime system and to obtain a preliminary design for the placement of the lifetime detectors. Experiments using both dummy samples and the encapsulated $\mathrm{Pu}$ sample were performed to determine the effects of background components and to optimize the detector-sample geometry in order to obtain high quality data.

During these measurements we continued to refine our spectrographic technique. Initial data showed unambiguous features related to defects in the material but detailed analysis demonstrated that cleaner data and additional measurements were necessary to provide accurate values of defect parameters. Improved detectors and changes in detector and sample geometry resulted in spectra with lower contributions from backgrounds and artifacts and additional measurements were performed. Refinement of the experiment through geometric improvements and better detectors will continue and we are storing our samples in an archive for additional measurements.

A first measurement of aged $\mathrm{Pu}$ on two separate 21 year old, $\delta$ phase samples from the same source has been performed. The time distribution of the annihilation of implanted positrons is seen in the attached figure. Reduction of these data into annihilation rates is performed by least squares fit of a model of the spectrum that includes one or more separate annihilation rates, a background from uncorrelated gamma rays and the time resolution of the detector system. Analysis of the $\mathrm{Pu}$ data produces only two identified annihilation lifetimes. A single lifetime of $284 \mathrm{ps}$ is found from the $\mathrm{Pu}$ sample and a small contribution, $1 \%$, is 
identified by measurements of other materials as background from positrons annihilating in the wrapping of the detector system with a lifetime of $800 \mathrm{ps}$.

The results of the lifetime analysis show that all positrons are annihilating in the same condition. If all the positrons are trapped then the trap concentration must be of the order of 1 part per 1000 or higher. If two separate trapping defects such as single vacancies and voids were competing for the positron then we would see separate contributions in the lifetime spectra from the two defects. Also the relatively short value of 284 ps suggests that the traps are not large empty voids. So these data are showing a relatively strong concentration of defects with lifetimes too small to be empty voids.

To identify the annihilation state and the defect species in $\mathrm{Pu}$ requires knowledge of the annihilation rates for each kind of defect. Many of these values can be obtained by ab initio calculation. First calculations for the values of positron lifetimes were performed for defect free $\mathrm{Pu}$ and specific Pu defects. Defect free, $\delta$ phase $\mathrm{Pu}$ was predicted to have a lifetime of 139 ps and a single vacancy in an undeformed lattice 249 ps. Adding He to a vacancy will add electrons and lower the annihilation lifetime. Initial calculations for vacancies containing He were predicted to have annihilation lifetimes between 167 ps and 193 ps depending of the number and location of the He atoms. Comparison of these values to the analysis of our data at 184 ps suggests a high concentration of He stabilized defects in aged material. This conclusion must be regarded as tentative since several defect-He combinations remain to be calculated and we have not yet made verifying measurements of defect free material.

A high concentration of He stabilized vacancies or vacancy clusters is a necessary condition for void growth and swelling. Vacancies produced by the recoiling atoms during the radioactive decay are lost to recombination with interstitial atoms, grain boundaries, impurities and other microstructrual feature unless they are stabilized. All models of void swelling proceed from migration of vacancies that have been stabilized by He. Thus in 21 year old $\mathrm{Pu}$ we have found the precursor to void swelling but no evidence of voids in the samples measured. 


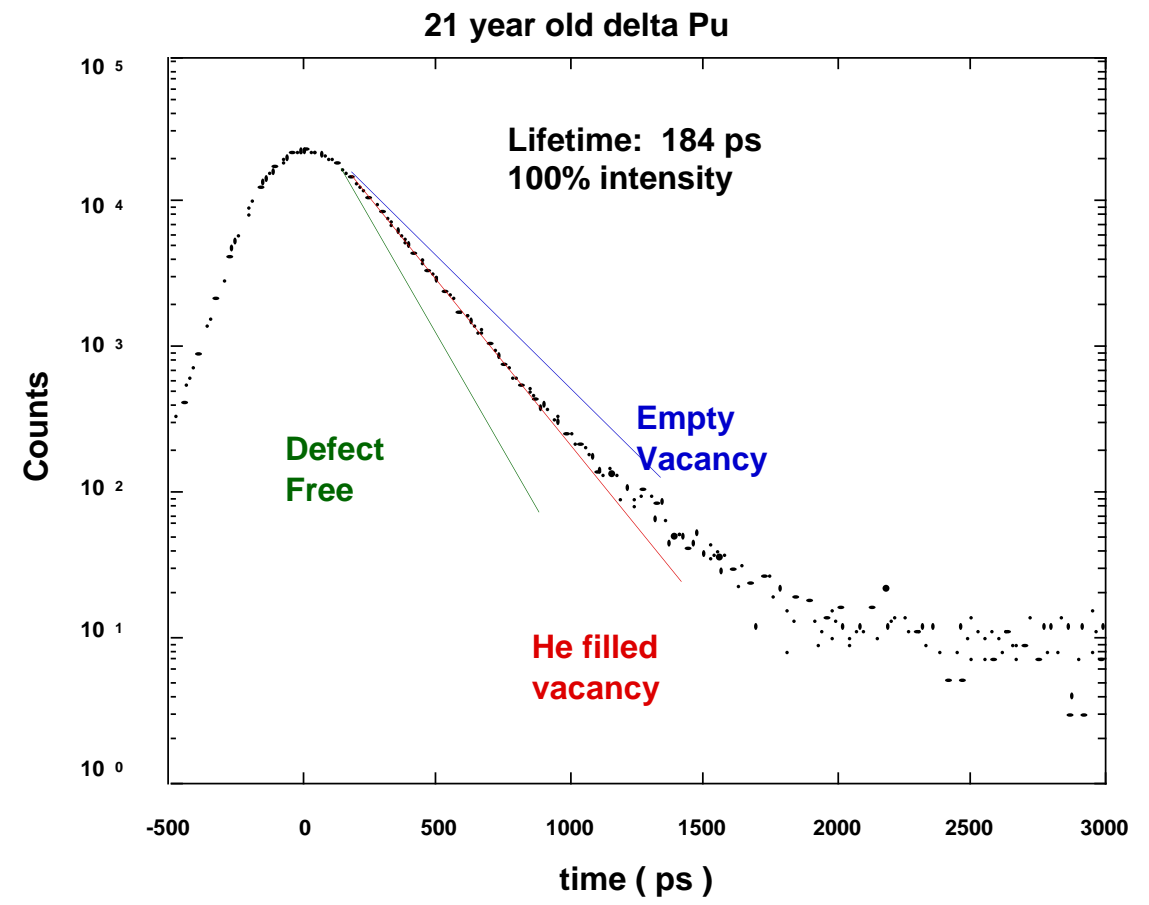

Figure 1. Time distribution of positron annihilations in aged $\delta$ phase $\mathrm{Pu}$. Inset lines are calculated annihilation rates for defect free material, vacancies and $\mathrm{He}$ filled vacancies. 


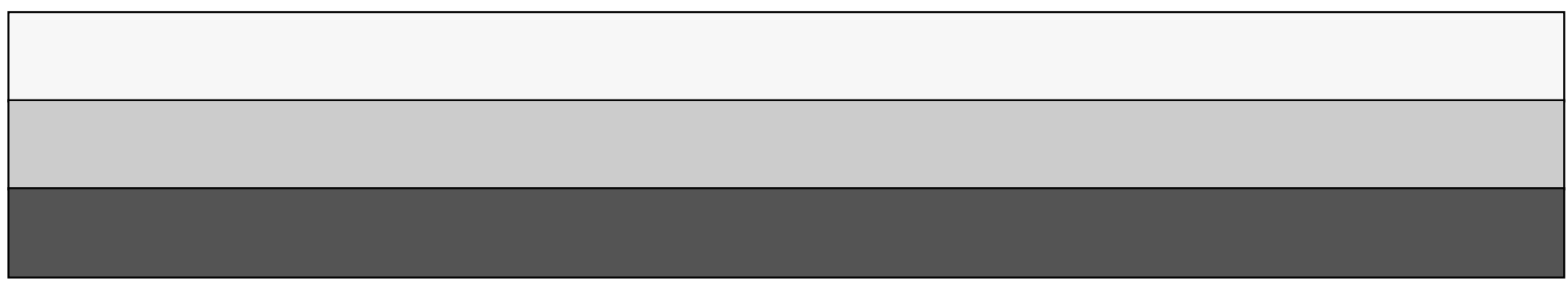

